\title{
Preface: Genome editing in plants
}

\author{
Paul Christou • Amit Dhingra - Inez H. Slamet-Loedin • Margarida Oliveira • \\ Supriya Chakraborty · Johannes Buyel • Eva Stoger • Stefan Schillberg • \\ Diego Orzaez $\cdot$ Hector Quemada
}

Accepted: 4 June 2021

(C) The Author(s), under exclusive licence to Springer Nature Switzerland AG 2021

Genome editing is a suite of technologies that allows the precise and efficient modification of DNA. This presents a significant advantage over the previous generation of transgenic technologies that rely on random DNA integration as well as the far less efficient gene targeting methods of the 1990s based on homologous recombination and the random mutations induced by chemicals or ionizing radiation. Genome editing was developed in the 2010s and has

P. Christou $(\bowtie)$

Universitat de Lleida-Agrotecnio CERCA Center, Lleida, Spain

e-mail: paul.christou@udl.cat

P. Christou

ICREA, Catalan Institute for Research and Advanced

Studies, Barcelona, Spain

A. Dhingra

Department of Horticulture, Washington State University, Pullman, WA, USA

I. H. Slamet-Loedin

International Rice Research Institute, Los Baños,

Philippines

M. Oliveira

Instituto de Tecnologia Química E Biológica António

Xavier, Universidade Nova de Lisboa, Oeiras, Portugal

S. Chakraborty

School of Life Sciences, Jawaharlal Nehru University,

New Delhi, India consolidated into three major platforms that use designer nucleases to introduce targeted double-strand breaks (DSBs) into genomic DNA, as well as a related set of technologies that modify specific bases. The targeted introduction of DSBs promotes endogenous repair mechanisms, primarily non-homologous end joining (NHEJ), which is an error-prone pathway that primarily generates small insertions and deletions (indels) at the target site, often causing loss-of-

\author{
J. Buyel \\ Fraunhofer Institute for Molecular Biology and Applied \\ Ecology IME, RWTH Aachen University, Aachen, \\ Germany \\ E. Stoger \\ Department for Applied Genetics and Cell Biology, \\ University of Natural Resources and Life Sciences, \\ Vienna, Austria \\ S. Schillberg \\ Fraunhofer Institute for Molecular Biology and Applied \\ Ecology IME, Aachen, Germany \\ D. Orzaez \\ Instituto de Biologia Molecular Y Celular de Plantas \\ (IBMCP), Universitat Politecnica de Valencia- CSIC, \\ Valencia, Spain \\ H. Quemada \\ Department of Biological Sciences, Western Michigan \\ Univ, Kalamazoo, MI, USA
}


function mutations when the target is within a gene. The provision of a repair template can stimulate homology-dependent repair (HDR) at a lower frequency, allowing more precise mutations (substitutions, resulting in allele replacement, or even the insertion of new sequences). In contrast, the nucleobase editing mechanisms modify individual bases in situ and stimulate base excision repair and mismatch repair mechanisms to generate substitutions.

In addition to advances in precision and efficiency, genome editing has above all achieved advances in convenience and versatility, especially the CRISPR/ Cas platform, which can be used for canonical genome editing via the NHEJ and HDR pathways, or can be modified to incorporate enzymes that target specific nucleobases, or even allow the targeted modification of epigenetic markers. The mechanics of the CRISPR/ Cas platform can also be customized extensively to control the specificity of the components, the preferred target sites, the activity of the Cas nuclease in terms of DSB structure and position, nickase activity, or even DNA binding without cleavage, and the interaction with other components such as the transcriptional apparatus, making even more sophisticated approaches such as prime editing possible. Genome editing can be applied in any species, explant or cell type as long as the components can be delivered and/or expressed, and this has led to an explosion of applications, particularly in medicine and agriculture. This special issue of Transgenic Research includes 11 review articles written by experts in the field who have captured the scope and achievements of genome editing in the context of plant science and agriculture, looking at the technological basis as well as applications in different contexts.

In the first article, Ghogare et al. (2021) comprehensively address the available methods for "Genome editing reagent delivery in plants", discussing not only conventional methods based on dedifferentiated plant cells and protoplasts but also in planta methods involving the editing of germline cells or seeds. The authors describe successful methods for the delivery of reagents to plant cells and whole plants as well as challenges that hinder the use of genome editing for rapid crop improvement.

Schaart et al. (2021) in their article "Genome editing of polyploid crops: prospects, achievements and bottlenecks", focus on the special challenges presented by polyploid crops. Conventional breeding as well as many molecular breeding approaches are complex in polyploid crops because there are multiple alleles for each gene. CRISPR/Cas has been especially useful for targeted mutagenesis in polyploids because the guide RNA (gRNA) can be designed to match more or less conserved regions and thus target one, multiple or even all alleles and/or copies of a gene. Recent examples of heritable chromosomal rearrangements and their future prospects for the acceleration of breeding programs are also discussed.

Čermák's (2021) article, "Sequence modification on demand: search and replace tools for precise gene editing in plants", describes how genome editing provides a faster and more precise way to create new variation in the gene pool by allele replacement. He describes recent developments in precision editing involving the regulation of repair pathway choices or alternative mechanisms of DNA repair. He discusses novel genome editing tools that are independent of DSB repair or even independent of host DNA repair processes altogether, such as base editing and prime editing. Allele replacement has the potential to overcome the problem of genetic linkage to negative alleles in conventional breeding, thus accelerating the introgression of important traits into plants. Remaining bottlenecks are highlighted as well as future prospects and expectations.

The addition of functional domains such as epigenetic effectors and chromatin modifiers to the CRISPR/Cas ribonucleoprotein complex allows the introduction of targeted epigenomic modifications in plants. Selma and Orzaez (2021) in their article "Perspectives for epigenetic editing in crops", review various technologies for programmable epigenomic editing, including the regulation of gene expression. The authors examine a number of important agronomic traits known to be under epigenetic control and discuss examples of epigenetic effectors in plants including DNA methylases/demethylases, histone acetylases/deacetylases and their associated marks. The review also discusses the use of these tools in crop breeding.

In the last three decades, plants have been developed as production vehicles for recombinant proteins and small molecules with applications in medicine, industry or consumer markets such as cosmetics. In their article "Targeted genome editing of plants and plant cells for biomanufacturing", Buyel et al. (2021) consider the applications of genome editing in the field 
of plant molecular farming, which has yet to be embraced broadly by industry despite its many advantages, mostly due to regulatory uncertainty, limited information on process cost structures, and limited capacity. In addition, plant-based production needs to meet the strict requirements of industry-scale manufacturing in terms of productivity, quality control and regulatory acceptance. The article discusses how genome editing technologies could improve plants as biomanufacturing platforms and also illustrates how genome editing and conventional transgenesis technologies can complement each other to establish host plants for such applications.

Genome editing can also be used to protect plants against pests and diseases. Rato et al. (2021) in their article "Genome editing for resistance against plant pests and pathogens", discuss recent reports on the editing of plant susceptibility genes and pathogen virulence factors. The article offers an overview of fundamental and proof-of-concept investigations demonstrating the potential of genome editing to address such constraints in agriculture. It also shows how genome editing can be combined with other technologies to develop new solutions for environmentally-friendly and sustainable plant protection.

Improved cereal crops with higher yields and more resilience to environmental stress have contributed to global food security since the Green Revolution, and genome editing could be the basis for the next Green Revolution, targeting important agronomic and quality traits in cereals directly. Matres et al. (2021) in their article "Genome editing in cereal crops-an overview", evaluate cereal traits that have been modified using genome editing, including traits that mitigate the effects of climate change and those conferring tolerance to biotic or abiotic stress, higher yields, optimal plant architecture, improved grain quality and nutritional content, and safer products. The review not only covers the latest applications of genome editing for trait improvement in cereals, but also discusses technical limitations and regulatory challenges that must be overcome for the technology to make an impact on global agriculture.

Genome editing has also provided new opportunities for trait enhancement in fruit trees, ornamentals, and other specialty crops. Ramirez-Torres et al. (2021) in their article "Genome editing in fruit, ornamental, and industrial crops" review the applications of genome editing in such crops to enhance fruit ripening, increase stress tolerance, modify plant architecture, control flower development, and enhance the accumulation of desirable metabolites, among other commercially important traits. Targeted gene editing is particularly useful to accelerate trait improvement in tree and shrub species with long juvenile phases.

Genome editing tools are evolving rapidly, and recent developments include the discovery of alternative Cas nucleases and the development of engineered Cas variants that minimize PAM restrictions, offtarget effects and temperature sensitivity. Huang and Puchta (2021) discuss various CRISPR/Cas applications in plants, including precise genome editing via the HDR pathway, base editing and prime editing. They also consider more recent applications for heritable chromosomal rearrangements (such as inversions and translocations), and strategies for tissue culture-free genome editing, which overcome the transformation bottleneck in many recalcitrant crops and genotypes. The article "Novel CRISPR/Cas applications in plants-from prime editing to chromosome engineering" not only provides the reader with an overview of the latest developments in plant genome editing, but also with decision-making tools for fundamental science questions and applications.

Entine et al. (2021) in their article "Regulatory approaches for genome edited agricultural plants in select countries and jurisdictions around the world", give an account of the regulatory landscape governing genome editing in agriculture and food products in different countries and regions. Agricultural products developed using genome editing must be governed by science-based safety regulations. This is particularly true for commodity crops, considering the proportion of such crops in international trade, and the prospect of their being subject to multiple, inconsistent and nonscience based regulations as they traverse different jurisdictions.

The versatility and broad applications of the CRISPR/Cas9 system and its variants has unsurprisingly led to a proliferation of patents and patent applications for various uses of the technology. Azzam-Jefferson et al. (2021) in their article "Mapping innovation trajectories of CRISPR/Cas9 technology using a dynamic and granular Lens toolbox" allow the reader to navigate the complex intellectual property landscape of CRISPR/Cas9 technology. Patents related to genome editing may be for non- 
commercial research or the development of products such as reagents, equipment, toolkits and vectors. This rich patent landscape exists in part because none of the broad patents covering CRISPR/Cas9-related inventions give exclusive rights to their owners for the exploitation and commercialization of the technology.

Together, these review articles cover multiple aspects of the genome editing landscape, including scientific and technical characteristics, applications in different agricultural and pharmaceutical contexts and as applied to different types of crops, and the regulatory and knowledge management issues that must be navigated to use genome editing technologies for research and/or commercial purposes. They discuss the technological breakthroughs that have been achieved with genome editing technologies but also the hurdles that must still be overcome and prospects for the application of these technologies in the future.

\section{References}

Buyel JF, Stöger E, Bortesi L (2021) Targeted genome editing of plants and plant cells for biomanufacturing. Transgenic Res. https://doi.org/10.1007/s11248-021-00236-z

Cermak T (2021) Sequence modification on demand: search and replace tools for precise gene editing in plants. Transgenic Res. https://doi.org/10.1007/s11248-021-00253-y

Entine J, Felipe MSS, Groenewald JH et al (2021) Regulatory approaches for genome edited agricultural plants in select countries and jurisdictions around the world. Transgenic Res. https://doi.org/10.1007/s11248-021-00257-8

Ghogare R, Ludwig Y, Bueno GM et al (2021) Genome editing reagent delivery in plants. Transgenic Res. https://doi.org/ 10.1007/s11248-021-00239-w

Huang TK, Puchta H (2021) Novel CRISPR/Cas applications in plants: from prime editing to chromosome engineering. Transgenic Res. https://doi.org/10.1007/s11248-02100238-X

Jefferson OA, Lang S, Williams K et al (2021) Mapping CRISPR-Cas 9 public and commercial innovation using the lens institutional toolkit. Transgenic Res. https://doi.org/ 10.1007/s11248-021-00237-y

Matres JM, Hilscher J, Datta A et al (2021) Genome editing in cereal crops: an overview. Transgenic Res. https://doi.org/ 10.1007/s11248-021-00259-6

Ramirez-Torres F, Ghogare R, Stowe E et al (2021) Genome editing in fruit, ornamental, and industrial crops. Transgenic Res. https://doi.org/10.1007/s11248-021-00240-3

Rato C, Carvalho MF, Azevedo C, Oblessuc PR (2021) Genome editing for resistance against plant pests and pathogens. Transgenic Res. https://doi.org/10.1007/s11248-02100262-x

Schaart JG, van de Wiel CCM, Smulders MJM (2021) Genome editing of polyploid crops: prospects, achievements and bottlenecks. Transgenic Res. https://doi.org/10.1007/ s11248-021-00251-0

Selma S, Orzáez D (2021) Perspectives for epigenetic editing in crops. Transgenic Res. https://doi.org/10.1007/s11248021-00252-z

Publisher's Note Springer Nature remains neutral with regard to jurisdictional claims in published maps and institutional affiliations. 